Pierre-Alain Duc, Jonathan Braine and Elias Brinks, eds.

\title{
A Structural NIR Analysis of the Interacting Pair of Galaxies KPG 404 (NGC 5394/95)
}

\author{
Margarita Valdez-Gutiérrez \\ Departamento de Astronomía, Universidad de Guanajuato, Apartado \\ Postal 144, 36000 Guanajuato, Gto, Mexico
}

Ivânio Puerari, Izbeth Hernández-López

INAOE, Calle Luis Enrique Erro \#1, 72840 Tonantzintla, Pue, Mexico

\begin{abstract}
We present near infrared observations in $\mathrm{J}, \mathrm{H}$ and $\mathrm{K}^{\prime}$ passbands of the interacting pair of galaxies KPG 404 (NGC 5394/95). We calculate total magnitudes, surface brightnesses and colour profiles. We present the growth curve to compare our photometry against earlier works in which circular aperture estimations were published. We also perform a structural (disk + bulge) analysis to get insight on the morphology of the pair. The disk+bulge fit shows that NGC 5394 (KPG 404A) is more compact than normal galaxies, while NGC 5395 (KPG 404B) is less concentrated. This suggests an interacting scenario in which NGC 5394 passes through the disk of NGC 5395 (a Cartwheel-type collision), rather than a passage as M51-type pairs.
\end{abstract}

Near infrared images are more suitable for a morphological analysis, because they map old stellar populations with little contribution of red giants (Rix \& Rieke 1993). In morphological studies based on $\mathrm{H} \alpha$ or optical images, the results will represent the morphology of a minority (and fast evolving) part of the disk mass. On the other hand if NIR images are used, the majority of the disk mass is being analyzed. Furthermore, the attenuation by dust is much smaller in NIR than in optical bands (Martin \& Whittet 1990). In this way, near infrared images trace the gravitational potential of the disk of galaxies.

We have analysed NIR ( $\mathrm{J}, \mathrm{H}$ and $\mathrm{K}^{\prime}$ ) images of the interacting pair of galaxies KPG 404 (NGC 5394/95). This work is part of a Bachelor thesis (HernándezLópez 2002). The images were taken at the $2.1 \mathrm{~m}$ telescope of the Observatorio Astronómico Nacional at San Pedro Mártir, Baja California, México, using the near-infrared camera CAMILA (Cruz-González et al. 1994).

Total magnitudes were calculated and their values for NGC 5394 are (J, $\left.\mathrm{H}, \mathrm{K}^{\prime}\right)=(11.05,10.20,10.11)$ and for NGC 5395, $\left(\mathrm{J}, \mathrm{H}, \mathrm{K}^{\prime}\right)=(9.53,8.78,8.73)$. The values of the colours for NGC 5394 are $\left(\mathrm{J}-\mathrm{H}, \mathrm{J}-\mathrm{K}^{\prime}, \mathrm{H}-\mathrm{K}^{\prime}\right)=(0.85,0.94$, 0.09 ) and for NGC 5395, $\left(\mathrm{J}-\mathrm{H}, \mathrm{J}-\mathrm{K}^{\prime}, \mathrm{H}-\mathrm{K}^{\prime}\right)=(0.75,0.80,0.06)$. No total magnitudes were found in the literature, but Joseph et al. (1984) and Bushouse \& Stanford (1992) have published circular aperture estimations. In Figure 1 we plot the growth curves for the galaxies, in which we compare against Joseph et al. (1984) and Bushouse \& Stanford (1992) determination. The mean difference between our values and those of Joseph et al. (1984) is 0.12. Comparing with 
Bushouse \& Stanford (1992), the agreement is much better (mean difference equals to 0.03$)$.
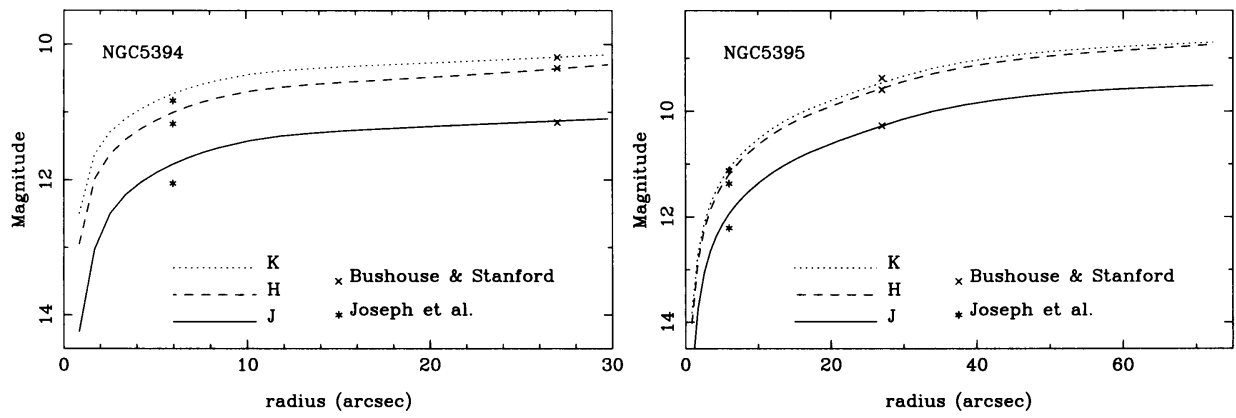

Figure 1. Growth curves.

We also calculate surface brightness profiles and we made a classical disk + bulge (exponential $+R^{-1 / 4}$ ) fit (see Figure 2). The values for $h$ and $R_{e}$ indicate that NGC 5394 is more compact than normal galaxies, while NGC 5395 is less concentrated. This suggests that the interaction between both galaxies is Cartwheel-type, with NGC 5394 crossing the disk of NGC 5395 (see also Puerari et al., in this issue).
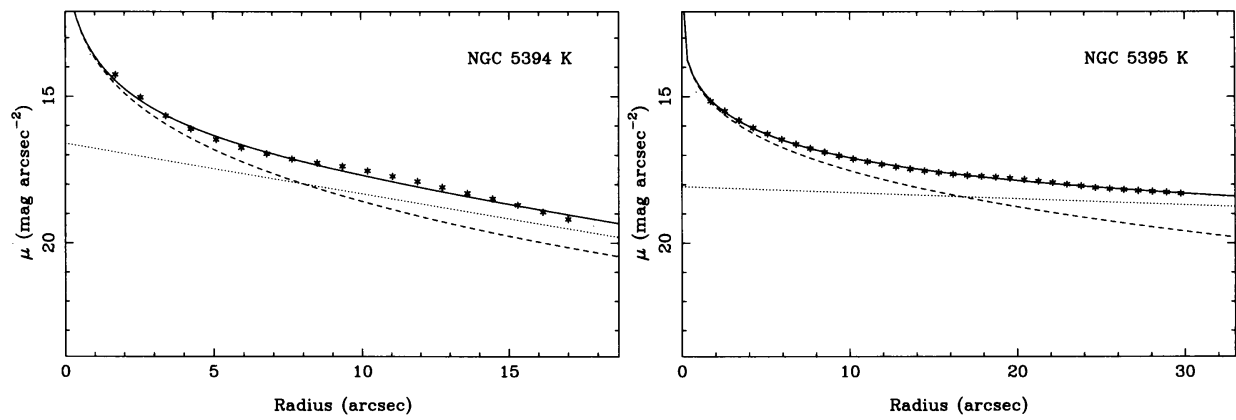

Figure 2. $\quad \mathrm{K}^{\prime}$ surface brightness profiles and disk + bulge fit.

Acknowledgments. MVG and IP thank the Mount Stromlo Observatory for its hospitality and financial support. This work is also supported by the Mexican Foundation CONACyT under project No. 36078-E.

\section{References}

Bushouse, H.A., Stanford, S.A. 1992, ApJS, 79, 213

Cruz-González, I., Carrasco, L., Ruiz, E., et al. 1994, In: Crawford D. L., Craine E.R. (eds.), Instrumentation in Astronomy VIII, Proc. SPIE 2198, 774

Hernández-López, I. 2002, BSc Thesis, BUAP, México

Joseph, R.D., et al. 1984, MNRAS, 209, 111

Martin, P.G., Whittet, D.G.B. 1990, ApJ, 357, 113

Rix, H.-W., Rieke, M.J. 1993, ApJ, 418, 123 